\title{
Design of the Human Body Detection-type Digital Door Lock using 12.8Ø Magnetic Field Coil
}

\author{
In-Sang Lee ${ }^{1}$ and Je-Ho Song ${ }^{1}{ }^{*}$ \\ ${ }^{1}$ Dept. of IT Applied System Engineering, Chonbuk National University, \\ 567Baekje-daero, Deokjin-gu,Jeonju-si,Jeollabuk-do(54896), Korea \\ ${ }^{1}\{$ wifizone_songjh\}@jbnu.ac.kr
}

\begin{abstract}
In this paper, we design of the human body detection-type digital door lock using $12.8 \emptyset$ magnetic field coil. In contract with digital door lock that are used nowadays, existing serial number entering buttons, lighting, number cover, corresponding PCB, exterior case, and data delivery cables have been deleted and are only composed of control ON/OFF power switches and emergency terminals. When the magnetic field coil substrates installed inside the inner case detects electric resistance values delivered from the opposite side of the $12 \mathrm{~mm}$ interval exterior contacting the glass body part, corresponding induced current flows. At this time, the magnetic field coil takes role as a sensor when coil frequency of the circular coil is transformed. The magnetic coil as a sensor detects change of oscillation frequency output before and after the body is detected. It is than amplified larger than 2,000\%, transformed into digital signals, and delivered to exclusive software to have the role of comparing and searching embedded data. As result of experiment, detection time followed by touch area of body standard to $12.8 \emptyset$ magnetic field coil was $30 \%$ contrast 0.08 sec and $80 \%$ contrast $0.03 \mathrm{sec}$ in which detection distance was $13.4 \mathrm{~mm}$ to show the best level.
\end{abstract}

Keywords: Coil, Door lock, Detection-type, Frequency, Magnetic field

\section{Introduction}

Digital door locks for tempered glass door are mainly installed at the doors of show window, office, store and the like on main street. And in case of having a slab structure without the eaves that can keep rain.snow off the doors, countermeasures against rain. snow are keenly required.

In case of conventional Korean products, damage is prevented by additionally purchasing and installing an extra plastic waterproof case. However, there is large inconvenience at ordinary times. And various side effects such as economic loss, deterioration in external appearance, and damp are produced. So, frequent A/S is caused[1].

In particular, a problem of being unable to use door locks without worry occurs because door locks are frequently destroyed by unidentified persons, and an illegal attempt to unlock doors is made where they are located on the crowded main street, or the outdoor case having an important identification input data function is easily accessible. Besides, conventional digital door lock products are too complicatedly composed of the outdoor case having a security code input function and the indoor case where a mechanism is actuated. Accordingly, 200 tons of zinc material is consumed for manufacturing digital door lock products every year, and a huge amount of aluminum material of 350 tons is consumed for manufacturing the case.

Received (October 6, 2017), Review Result (December 19, 2017), Accepted (January 18, 2018) 
In this paper is intended to carry out design of human body detection-type digital door lock using a $12.8 \varnothing$ magnetic coil without the outdoor case in order to get rid of that problem.

This digital door lock is installed in the entrance door made of tempered glass transmitting electromagnetic waves. Once an input device consisting of magnetic field coils is inserted into the indoor case, a human magnetic field passes through the glass door with a thickness of $12 \mathrm{~mm}$ and sends its own secret code to the input device. Using this technology, user can enter a password of the locking system in the glass door $[2,3]$.

This system maximizes security, which is the key of detection systems, since this does not require an outdoor case that is an indispensable component of the existing digital door locks and an indoor case replaces this, instead. Besides, malfunction caused by snow and rain is fundamentally prevented, the use of necessary raw materials decreases by $50 \%$, and wasted PCB and phenol, which bring about pollution drastically decline. By improving most of the existing weaknesses like this, it is expected that a large quantity of the existing products would be replaced and it would lead to ripple effects, such as the reorganization of the digital door lock market for tempered glass doors.

\section{Outline of technology}

It is technology of sending identification security code by making the magnetic field of human body passing through glass door of $12 \mathrm{~mm}$ in thickness from the outside of room, having a built-in input device composed of magnetic coil in the indoor case of digital door lock installed at tempered glass door.

Also, an outdoor case, which is an indispensable component of the existing products is not needed, because this is designed to enter a password of the locking system in the glass door and user does not need to install a separate plastic tempered case additionally for preventing this from snow and rain.

Figure 1 shows the schematic diagram of human body detection-type digital door lock using $12.8 \emptyset$ magnetic field coil.

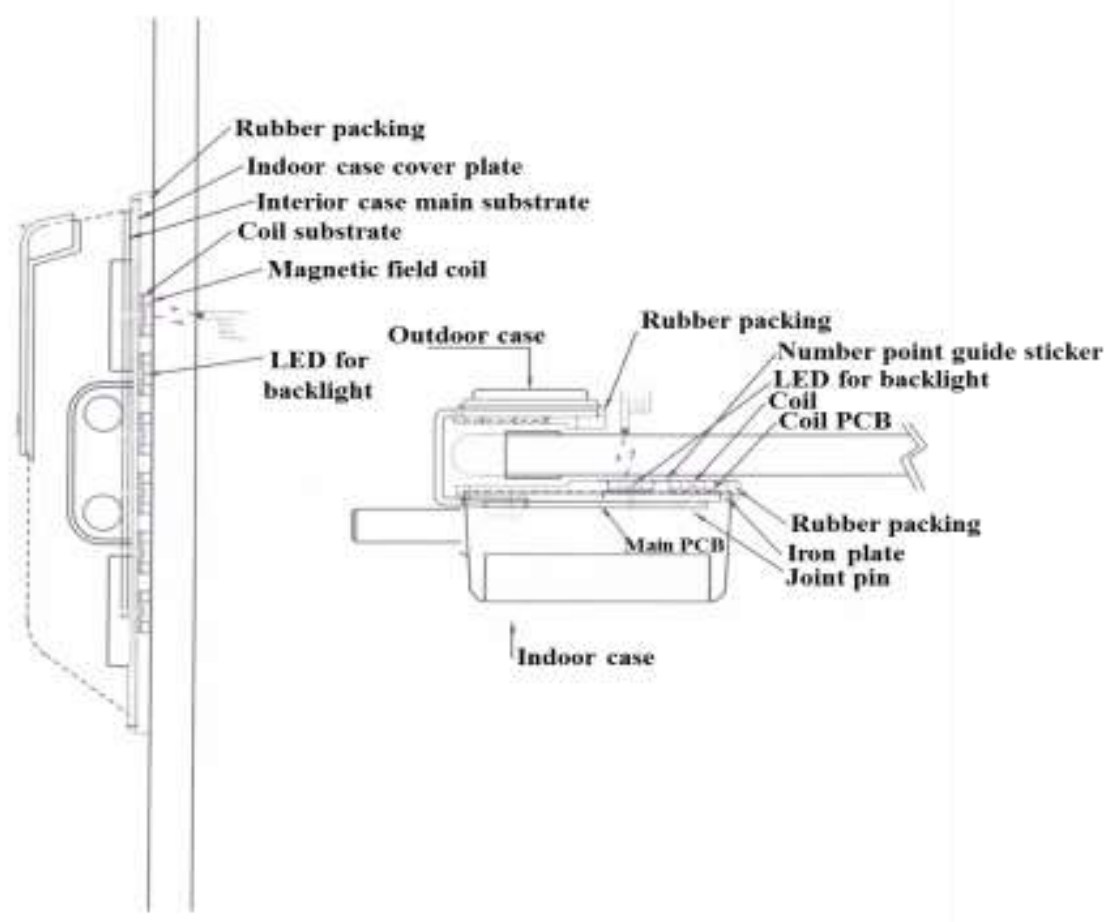

Figure 1. Schematic diagram of human body detection-type digital door lock using $\mathbf{1 2 . 8} \varnothing$ magnetic field coil 


\section{Human Body Detection-type Digital Door Lock Design using 12.8Ø Magnetic Field Coil}

Basically, a digital door lock is composed of an outdoor case, which is a data input device for a release of locking and an indoor case that performs physical functions. In this study, most parts and functions of the outdoor case are applied to the indoor case to implement basic functions of the locking device and the indoor case has a simple form without most structures and parts, except a bracket that is necessary for installation. Also, the outdoor case only consists of an ON/OFF switch for operation and an emergency terminal, except a secret number input button, lighting, a protective cover, a PCB, an outer case, and a data transmission cable[4].

It plays a role of sensor as the frequency of the corresponding circular coil changes after electrical resistance value transmitted from the outside of room on the opposite side at a distance of $12 \mathrm{~mm}$ is detected, in case of directly inputting the security code of door lock on the glass door[5].

\subsection{Block Diagram}

Figure 2 shows a block diagram of human body detection-type digital door lock using $12.8 \varnothing$ magnetic field coil.

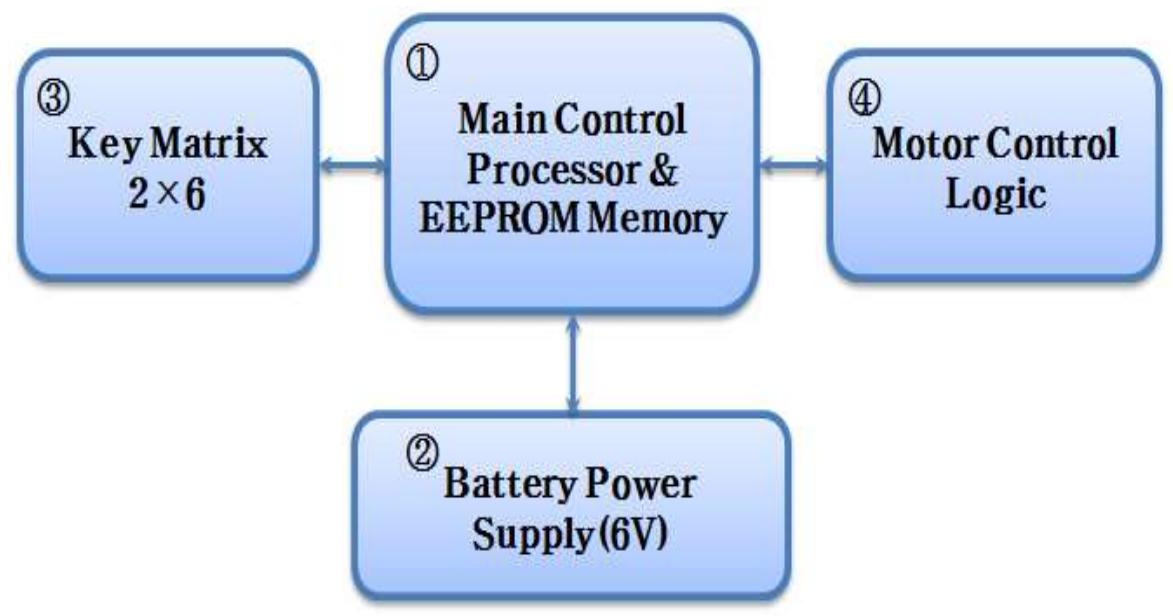

\section{Figure 2. Block Diagram of Human Body Detection-type Digital Door Lock using 12.8Ø Magnetic Field Coil}

Main Control Processor is the main control circuit to save different passwords and control-related data in the EEPROM, which is a non-volatile memory and to control all the functions using a storing control program.

Battery Power Supply is a circuit to supply batter power for operation of the system. Using four batteries, this operates at a power of $6 \mathrm{~V}$ and $1 \mathrm{~A}$ and for system output, this is equipped with a low-power LDO circuit.

Key Matrix consists of RLC oscillation circuits to detect twelve number keys in the back of the $12 \mathrm{~mm}$ tempered indoor glass and this oscillation circuit is to recognize a glass touch. As a circuit to recognize and control the button accurately by remedying the weakness of the current capacitance system, this is programmed to recognize a key touch through twelve 16-bit Compare and Counter Blocks in the main control circuit and performs the most important function, among the circuits of the glass door lock system.

For minimizing operation power of the system, also, Power Down Control automatically minimizes and saves power when there is no normal key touch within a 
setting time after the main circuit is invoked. For extending the operation hours of the system as long as possible, Power Auto Save is provided.

Motor Control Logic is a motor control circuit. Using two PNP-type Power TRs, two NPN-type Power TRs, and two switching TRs, this performs forward control and reverse control[5,6].

A batter input voltage of $5 \mathrm{~V}$ to $6 \mathrm{~V}$ is directly supplied and for motor control, residual capacity of the battery is calculated and the time to replace the battery is decided using 10-bit internal ADC. Once the final battery threshold voltage for stabilizing the system is detected, an alarm to replace the battery is triggered and a regular alarm is triggered until a proper action is taken.

\subsection{Magnetic Field Coil}

A current responding to micro resistance of human body is induced in the corresponding coil in case of grounding the human body (finger) to the outside coil point after applying a voltage of $\mathrm{DC} 6 \mathrm{~V}$ (four $1.5 \mathrm{~V}$ batteries) to the magnetic coil. Impedance is increased and inductance component is decreased by the electromagnetic coupling of magnetic coil, which occurs due to this induced current. Therefore, in case a voltage applied to the coil is $5.2[\mathrm{~V}], 8.7[\Omega]$ of resistance in the magnetic coil at the distance of $12 \mathrm{~mm}$ occurs due to finger[7-9].

The coil sensor detects a change in output oscillation frequency before and after detecting the human body, and then it is amplified by $2,000 \%$, and is converted to digital signal and is combined, and then is sent to software for exclusive use, and is compared with built-in fixed data, and a search is made.

The time to recognize a magnetic field depends on the habits of users when the human body(finger) touches the glass and it is hard to sense and identify an accurate amount of induced currents when checking the moment users start to press. Therefore, this system senses the moment when the human body is separate from the glass and the sensing time per area of the human body touching the glass is as follows.

Table 1. Detection time per field of finger

\begin{tabular}{|c|c|}
\hline Field & Detection time \\
\hline $3 / 10$ & $8 / 100 \mathrm{sec}$ \\
\hline $6 / 10$ & $6 / 100 \mathrm{sec}$ \\
\hline $8 / 10$ & $3 / 100 \mathrm{sec}$ \\
\hline
\end{tabular}




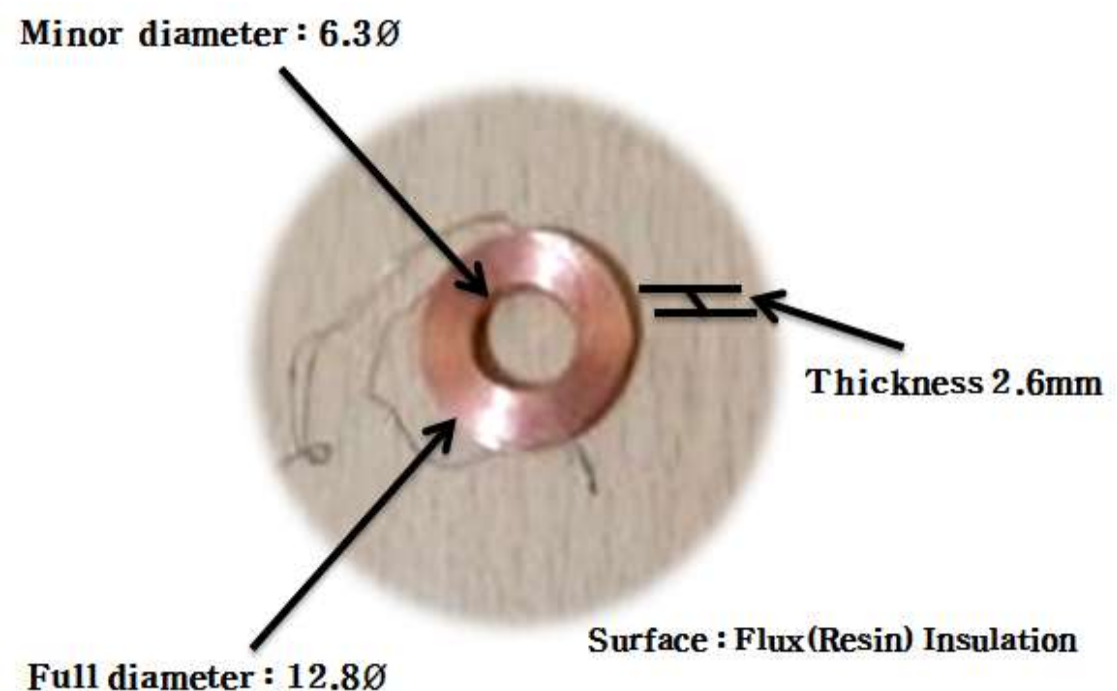

Figure 3. Magnetic field Coil

Figure 4 shows a circuit diagram of magnetic field coil.
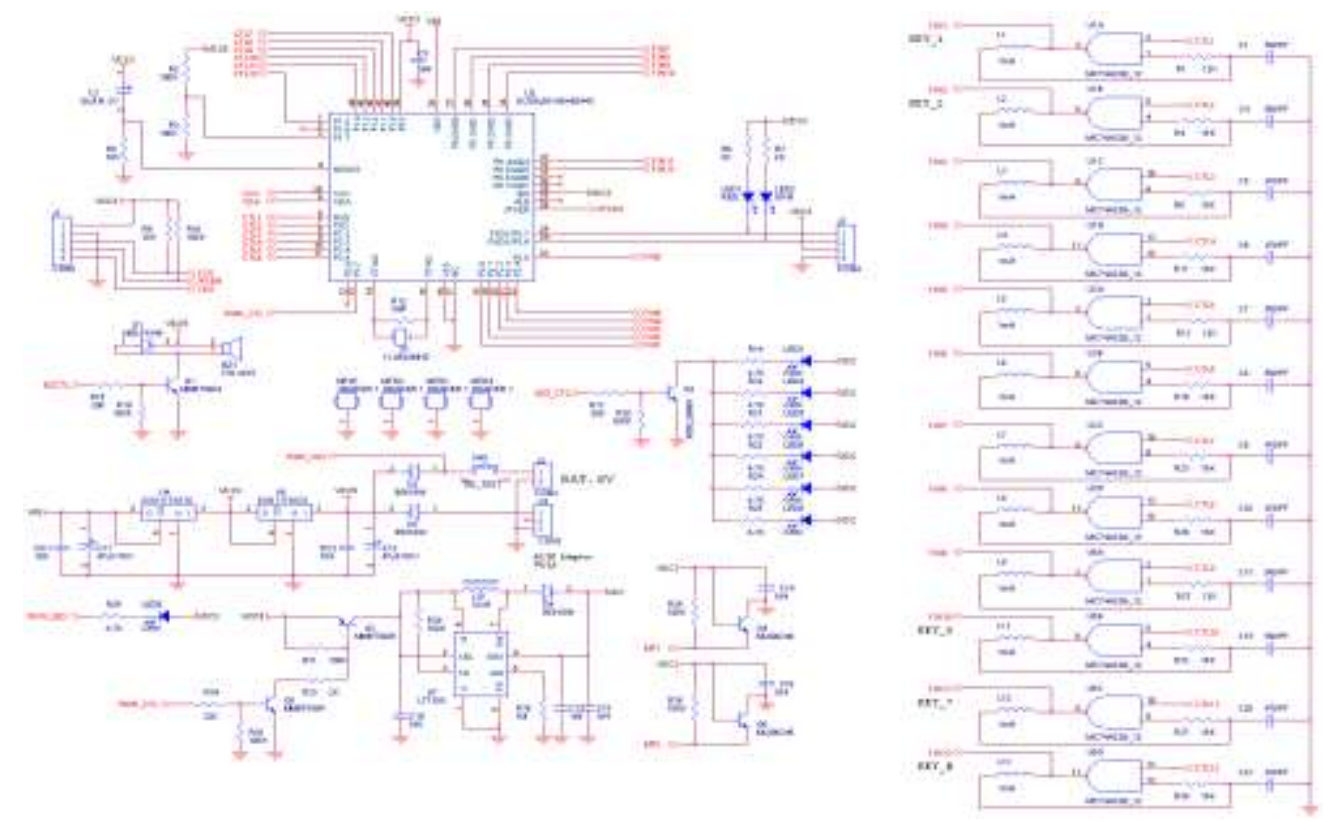

Figure 4. Circuit Diagram of Magnetic Field Coil

\subsection{Floor Plan of Human Body Detection-type Digital Door Lock}

Figures 5, 6, and 7 show the Floor plan views of indoor and outdoor cases, brackets, indoor and outdoor case rubber packing. Also, Figure 8 shows each part of a human body detection-type digital door lockthat was actually designed. 


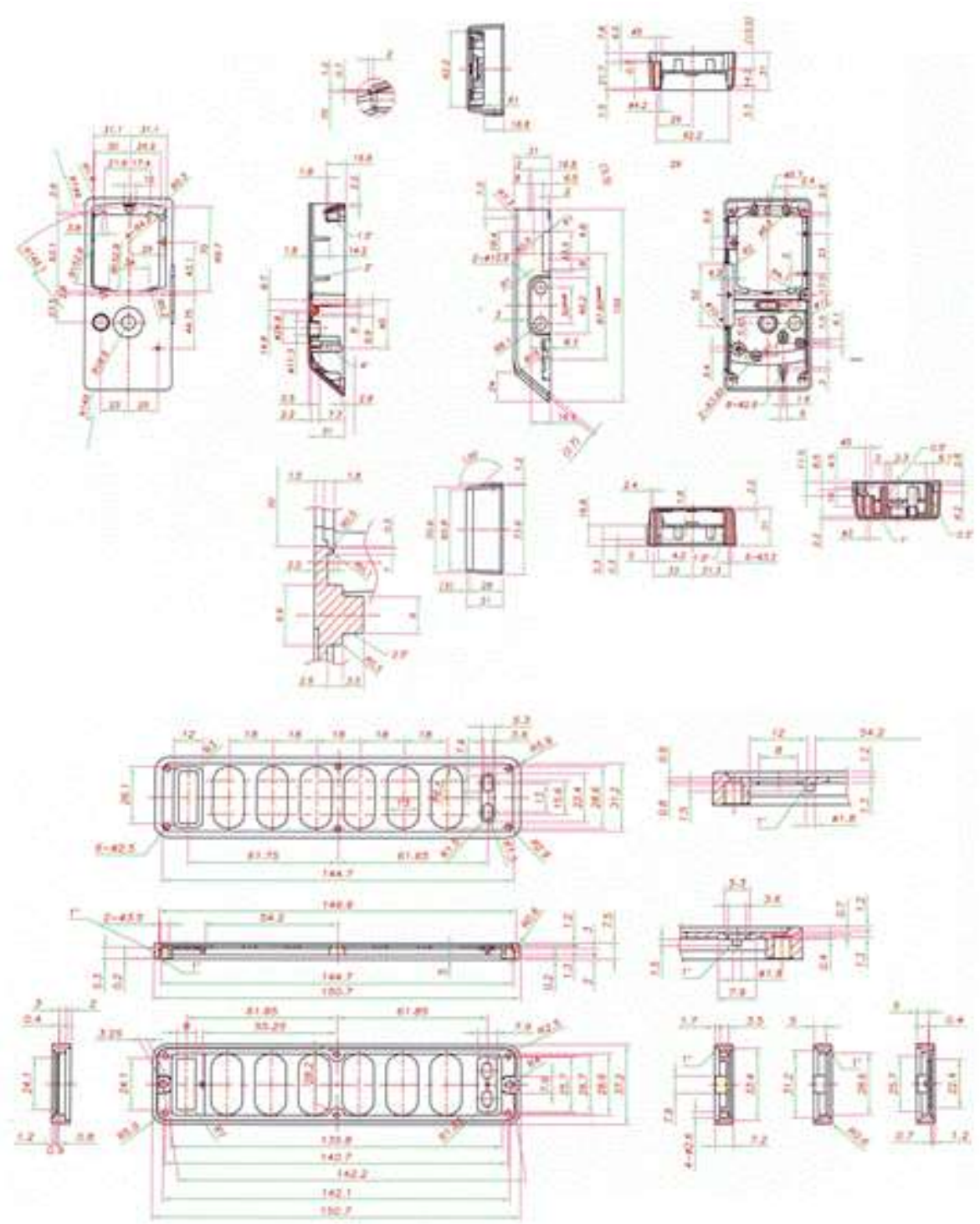

Figure 5. Floor plan of indoor and outdoor case
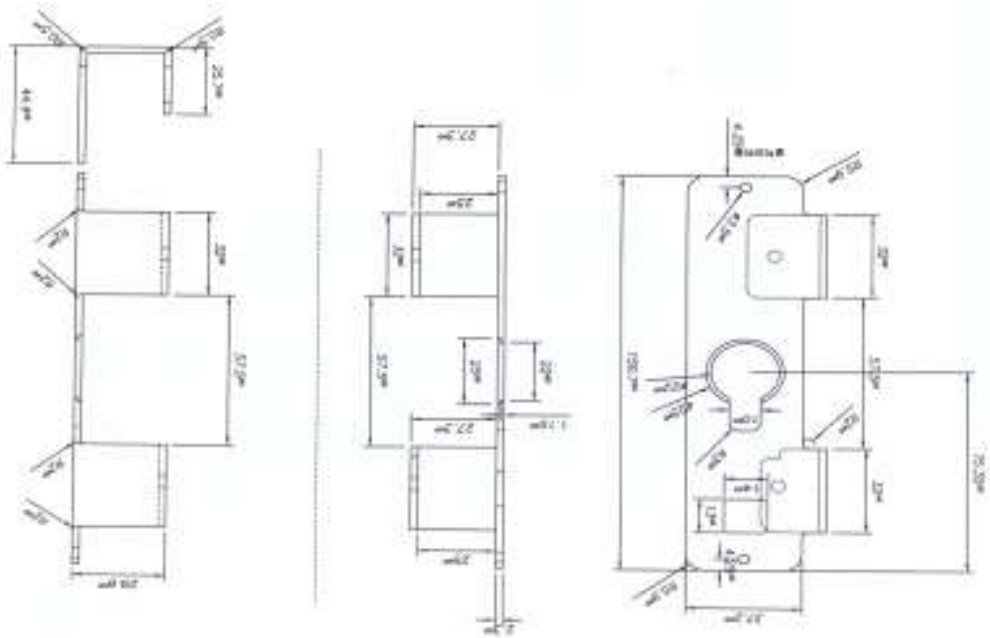

Figure 6. Floor Plan of Bracket 

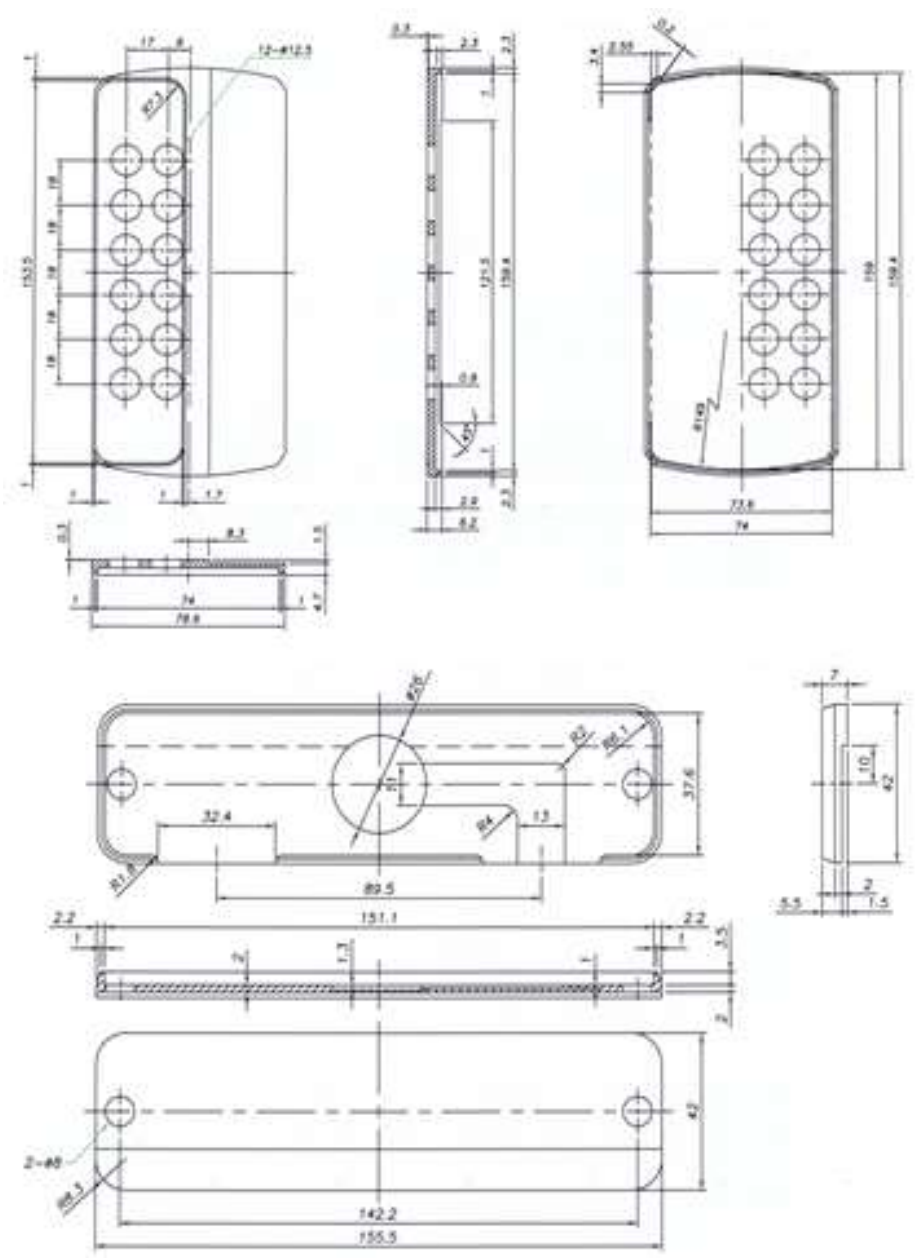

Figure 7. Floor Plan of Indoor and Outdoor Case Rubber Packing
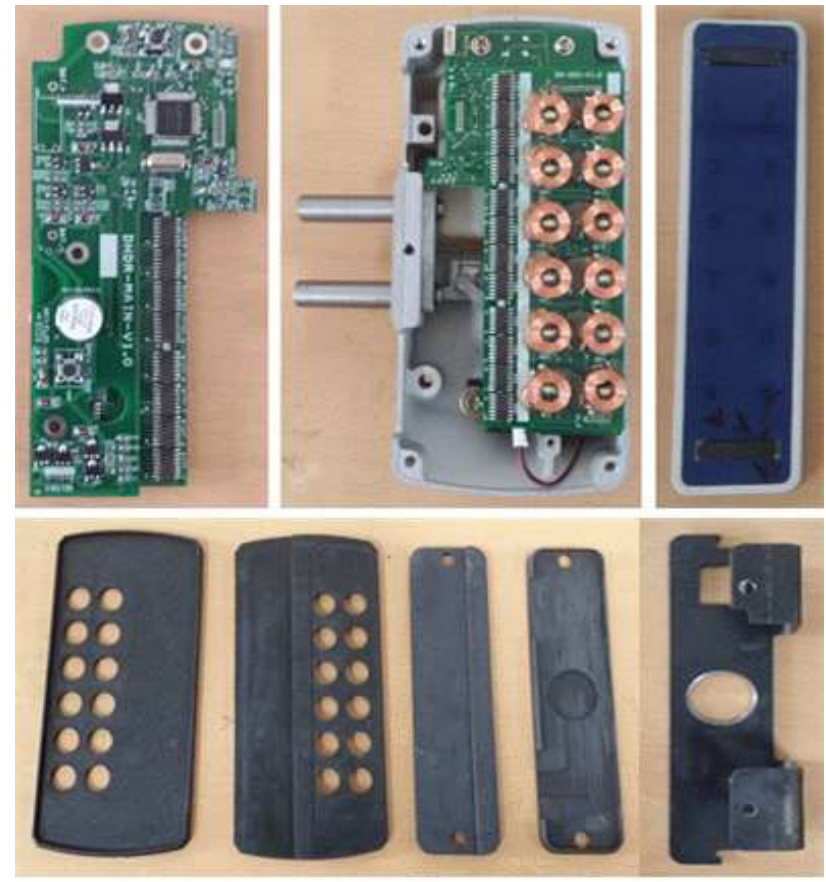

Figure 8. Floor Plan of Indoor and Outdoor Case Rubber Packing 


\subsection{Experiment Process of Human Body Detection-type Digital Door Lock}

Figure 9 shows a experiment process of human body detection-type digital door lock using $12.8 \varnothing$ magnetic field coil.
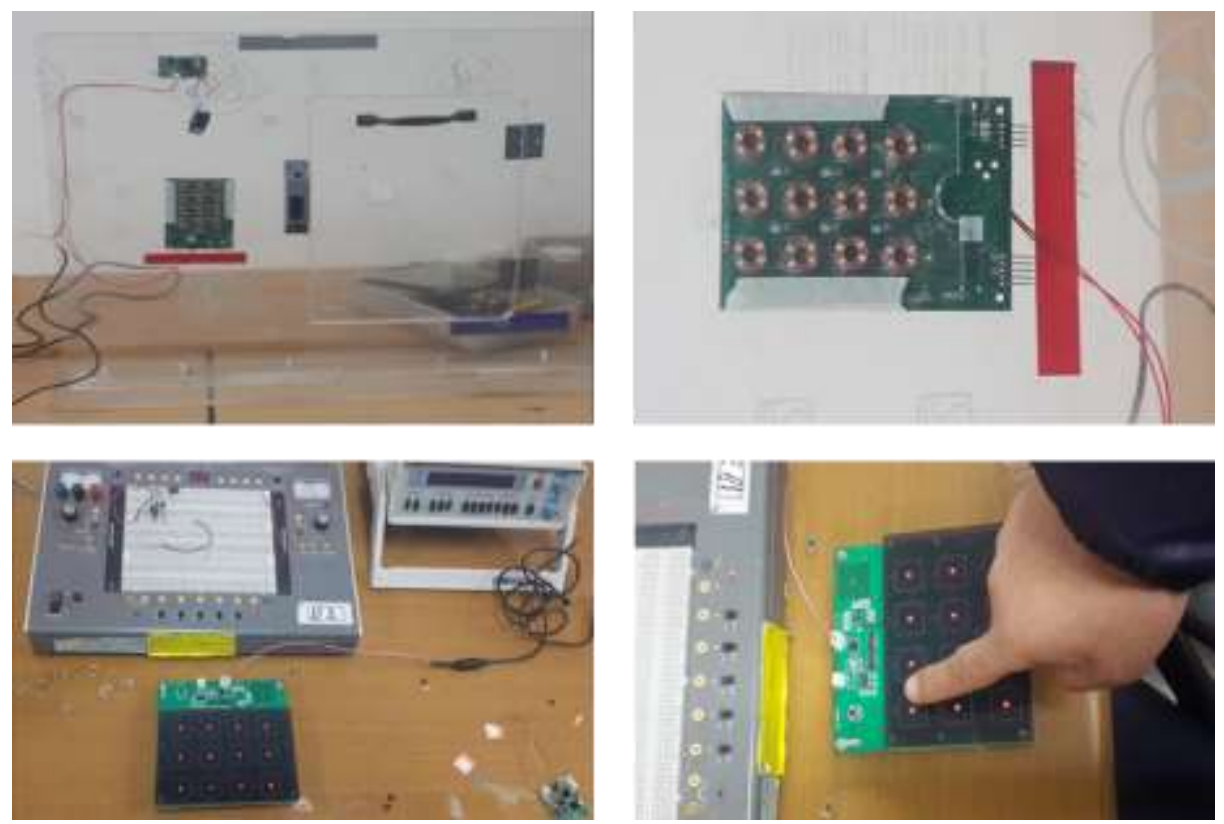

Figure 9. Experiment Process of Human Body Detection-type Digital Door Lock using 12.8Ø Magnetic Field Coil

In the experiment, the sensing time per touch area of the human body was $0.08 \mathrm{sec}$ for $30 \%$ and $0.03 \mathrm{sec}$ for $80 \%$, based on a magnetic coil of $12.8 \varnothing$ and according to the test report of an authorized institute, the sensing distance was $13.4 \mathrm{~mm}$, which was the highest level.

\subsection{Design of Human Body Detection-type Digital Door Lock}

The appearance of human body detection-type digital door lock using $12.8 \emptyset$ magnetic coil designed through this paper is shown in Figure 10[10].
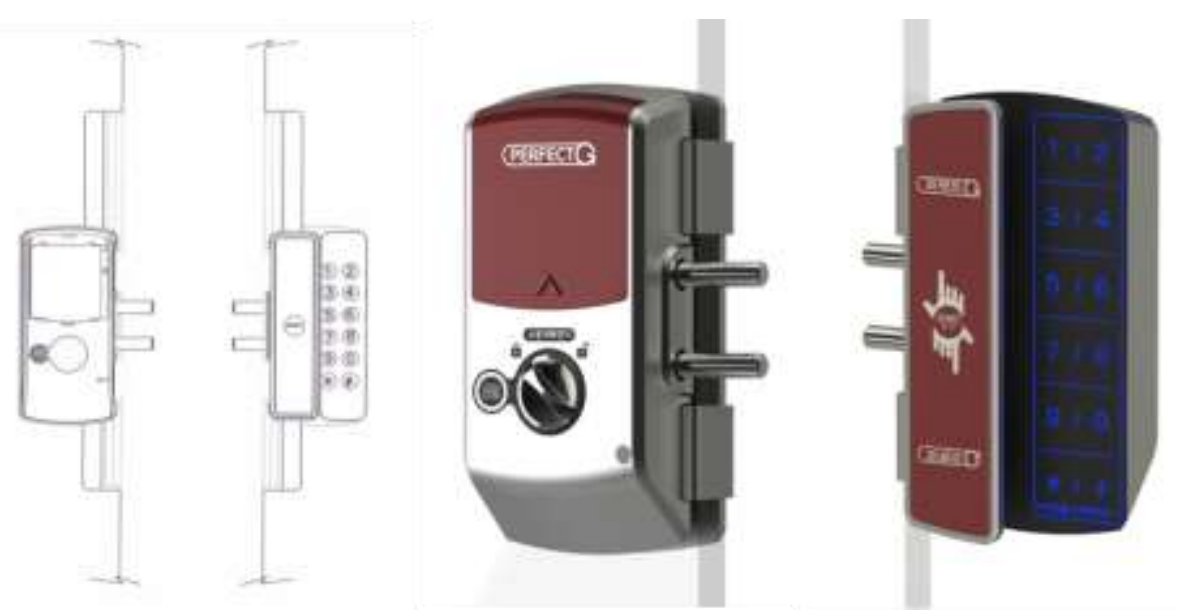

Figure 10. Human Body Detection-type Digital Door Lock using $12.8 \varnothing$ Magnetic Field Coil 


\section{Conclusion}

In case of the outdoor case of human body detection-type digital door lock using a $12.8 \emptyset$ magnetic coil, security number input button, lighting, protection cover, the corresponding pcb, enclosure, and data transmission cable was removed. And it was composed of only power supply ON/OFF switch and emergency terminal. And the indoor case was made to play a role of sensor as the frequency of the corresponding circular coil changed if electrical resistance value transmitted from the outside of room on the opposite side at a distance of $12 \mathrm{~mm}$ was detected in a condition where a magnetic coil board installed inside closely adhered to the body of glass door.

As result of research, detection time followed by touch area of body standard to $12.8 \varnothing$ magnetic field coil was $30 \%$ contrast $0.08 \mathrm{sec}$ and $80 \%$ contrast $0.03 \mathrm{sec}$ in which detection distance was $13.4 \mathrm{~mm}$ to show the best level.

Among the system components of conventional digital door lock products, the essential outdoor case becomes unnecessary. And the product can be composed of one part, i.e., indoor case. So, security, core of crime prevention device, is maximized. And the occurrence of malfunction due to snow.rain is totally prevented. And essential raw materials are reduced by $50 \%$. And the occurrence of waste PCB, phenol, and the like, pollutant, is drastically decreased. So, a large replacement demand will occur.

Therefore, in case of conventional digital door lock, there was a large loss at psychological and economic level. However, if the human body detection-type digital door lock using $12.8 \varnothing$ magnetic coil is designed, most of existing weak points will be gotten rid of due to functional and structural characteristics. So, it is expected that a ripple effect completely reshuffling the market of door lock for tempered glass door will be produced.

\section{References}

[1] Hee je kim, "Sensor Engineering”, Hongrung Publishing Company”, (2011).

[2] C. E. Lars1. The Institute of Electronics and Information Engineers, "Microwave Engineering", Cheongmoongak, (2003).

[3] Young wook Park, "Digital lock", Technology Commercialization laboratory, (2013).

[4] Jin tae Yoon, "Electromagnetic”, Taeyoung Publishing Company, (2010).

[5] Chang hwang Kim, "New Fundamentals of Electromagnetic Engineerings", Bokdoo Publishing Company, (2014).

[6] Young bum Jang, "Signals and Systems", Saengneung Publishing Company, (2010).

[7] Young kwon Sung, Byungmu Moon, "Physical Properties of Electronic Materials \& Devices", Sinsung Publishing Company, (2004).

[8] Ji hong Lee, "Microprocessor Application Experiment", GS-intervision, (2008).

[9] Il gu Ji, "Sensor Circuit Design and Experiment Training”, BM-sungandang, (2002).

[10] In chul Park, "A Study on the Design of Digital Door Lock", Korea Art Education Association, vol. 10, (2000), pp. 317-333.

\section{Authors}

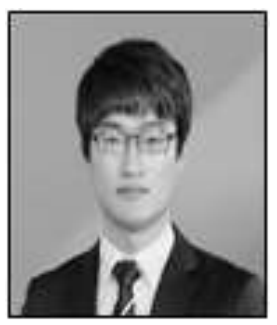

In-Sang Lee

August, 2016 : Master of IT Applied System Engineering, Chonbuk National University

September, $2016 \sim$ Present : Doctoral Program in IT Applied System Engineering, Chonbuk National University 


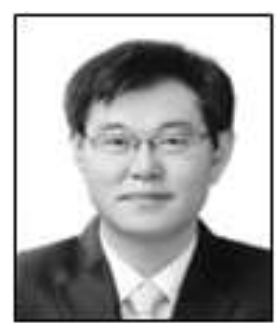

\section{Je-Ho Song}

March, $1996 \sim$ Present : Professor of IT Applied System Engineering, Chonbuk National University

January, 2011 present : Honam branch manager of the Korea Academia-Industrial cooperation Society, inc. 